Pacific Journal of Mathematics

IDEALS AND RADICALS OF SOME ENDOMORPHISM RINGS 


\title{
IDEALS AND RADICALS OF SOME ENDOMORPHISM RINGS
}

\author{
Jutta Hausen and Johnny A. Johnson
}

Let $R$ be the full ring of endomorphisms of a reduced abelian $p$-group $G$. A description is given, in terms of its action on $G$, of the Jacobson radical $J(R)$ of $R$ for the case that $G$ is sufficiently projective. Other ideals and radicals of $R$ and their relation to $J(R)$ are discussed.

1. The results. In order to study the structure of a ring $R$, one of the most important tools is the investigation of radicals. In this context, the following ideals are of interest. Throughout, the word ideal will mean two-sided ideal.

$N(R)$ : sum of all nilpotent ideals of $R$;

$B(R)$ : intersection of all prime ideals of $R$ (Baer radical);

$L(R)$ : sum of all locally nilpotent ideals of $R$ (Levitzki radical);

$K(R)$ : sum of all nil ideals of $R$ (Koethe radical);

$J(R)$ : sum of all quasi-regular ideals of $R$ (Jacobson radical). One has

$$
N(R) \subseteq B(R) \leqq L(R) \cong K(R) \cong J(R),
$$

and all of these ideals, with the possible exception of $J(R)$, are nil [4, pp. 193-197].

In this note we consider the case where $R=\operatorname{End} G$ is the full ring of endomorphisms of a reduced p-primary abelian group $G$. Our interest will be focused primarily on the Jacobson radical.

In [3] and [9], respectively, lower and upper bounds for $J($ End $G)$ where given, namely

$$
I(G) \subseteq J(\text { End } G) \subseteq H(G)
$$

which are defined as follows. Note that we write mappings to the right; throughout, $\lambda$ is a nonzero ordinal such that $p^{\lambda} G=0$. Then let $I(G)$ be the set of all $\varepsilon \in \operatorname{End} G$ for which there exists a finite sequence of ordinals $\sigma_{0}, \sigma_{1}, \cdots, \sigma_{k+1}$, where $k=k(\varepsilon)$, such that

$$
0=\sigma_{0}<\sigma_{1}<\cdots<\sigma_{k}<\sigma_{k+1}=\lambda
$$

and, for $i=0, \cdots, k, p^{\sigma_{i}} G[p] \varepsilon \subseteq p^{\sigma_{i+1}} G$. Let $H(G)$ be the set of all $\varepsilon \in$ End $G$ such that, for each nonnegative integer $n, p^{n} G[p] \varepsilon \subseteq p^{n+1} G$. Clearly, both $I(G)$ and $H(G)$ are ideals of End $G$. R. S. Pierce has shown that $J($ End $G)=H(G)$ if $G$ is torsion-complete, and $J($ End $G) \varsubsetneqq$ $H(G)$ if $G$ contains an unbounded direct summand which is a direct 
sum of cyclic groups [9, pp. 287, 288].

We will prove the following theorem. A reduced abelian $p$-group $G$ is called sufficiently projective if every countable subset of $G$ is contained in a totally projective direct summand of $G$.

THEOREM 1.3. If $G$ is sufficiently projective then $J($ End $G)=I(G)$.

Clearly, totally projective groups are sufficiently projective; the totally projective $p$-groups without elements of infinite height are just the direct sums of cyclic p-groups $[1$, p. $89 ; 11$, p. 251]. Thus, Theorem 1.3 generalizes Theorem 3.8 of [3] and the $p$-group version of (2.3) in [7].

As a consequence we obtain a characterization of the quasi-regular ideals of some endomorphism rings.

THEOREM 1.4. Let $G$ be sufficiently projective. Then the following properties of the ideal $I$ of End $G$ are equivalent.

(i ) I is quasi-regular.

(ii) I induces in $G[p]$ a nil ring of endomorphisms.

(iii) The restriction of $I$ to $G[p]$ is locally nilpotent.

Radicals of endomorphism rings other than the Jacobson radical, have received little attention in the past. If $G$ is unbounded then $R=\operatorname{End} G$ contains nil-ideals which are not nilpotent, for example $N(R)$ (see Corollary 2.5 below). This property characterizes the unbounded $p$-groups as the following result shows.

THEOREM 1.5. Let $R$ be the endomorphism ring of a reduced abelian p-group $G$. Then the following conditions are equivalent.

(i ) $N(R)$ is nilpotent.

(ii) $J(R)$ is nilpotent.

(iii) $J(R)$ is nil.

(iv) $J(R)=K(R)$.

( v ) $N(R)=B(R)=L(R)=K(R)=J(R)$.

(vi) $J(R)=N(R)$.

(vii) $G$ is bounded.

As pointed out in [5, p. 312], not all radicals that have been defined in the past are of equal importance. The Jacobson radical has been so successful since it seems to have a strong bearing on the structure of a ring. This is in coincidence with our investigations of endomorphism rings. The characterization of $J(\operatorname{End} G)$ in terms of its action on $G$ seems to be more tangible than the characterization of the other ideals of $R=\operatorname{End} G$ in (1.1). Moreover, in 
all cases such a characterization of $J(R)$ has been obtained [2, p. $27 ; 7$, p. $170 ; 9$, p. 287; Theorem 1.3 above], it was possible to completely describe $J(R)$ in terms of its action on $G[p]$. That this is not possible for the other ideals of (1.1), can be seen from the following result. Throughout, for subgroups $S$ and $T$ of $G, S \subseteq T$, Ann $(T / S)$ denotes the set of all $\varepsilon \in$ End $G$ such that $T \varepsilon \subseteq S$, and Ann $T=$ Ann $(T / 0)$. Clearly, if $S$ and $T$ are fully invariant, then Ann $(T / S)$ is an ideal of End $G$.

THEOREM 1.6. Let $R$ be the endomorphism ring of a sufficiently projective abelian p-group $G$ without elements of infinite height and let $J=$ Ann $G[p]$. Then

$$
J(R)=K(R)+J=L(R)+J=B(R)+J=N(R)+J .
$$

We conclude with a remark on various classes of $p$-groups.

The significance of the class of totally projective $p$-groups lies in the fact that it is the largest natural class of $p$-groups distinguishable by certain cardinal invariants [1, p. 100]. Recently, a number of new classes of $p$-groups have been introduced which properly contain the totally projectives and, for some of them, a classification by invariants has been obtained. Among those we mention Warfield's $S$-groups $[13,14]$ and the $C_{2}$-groups of Megibben and Wallace $[8,12]$ where $\lambda$ is an uncountable limit ordinal. However, for $p$-groups without elements of infinite height all of these classes coincide and reduce to the class of direct sums of cyclic groups which are the totally projective groups of length at most $\omega$. This is not the case for the class of sufficiently projective $p$ groups introduced in this article: there exist sufficiently projective $p$-groups of length $\omega$ which are not direct sums of cyclic groups and, hence, not totally projective $[1 ;$ p. 50].

2. The proofs. Throughout the following, $R=\operatorname{End} G$. We postpone the proof of Theorem 1.3 and start with a few observations on endomorphism rings of arbitrary reduced $p$-groups. As above, $\lambda$ is a nonzero ordinal such that $p^{\lambda} G=0$.

Lemma 2.1. The restriction of $I(G)$ to $G[p]$ is a locally nilpotent ring of endomorphisms of $G[p]$.

Proof. Note that $I(G)$ is the sum (and set union) of ideals whose restriction to $G[p]$ is nilpotent, namely

$$
I(G)=\bigcup_{\substack{\phi \subseteq \lambda \\ \phi \text { inite }}} I(\Phi)=\sum_{\substack{\phi \subseteq \complement \lambda \\ \phi \text { finite }}} I(\Phi),
$$


where, for $\Phi$ a finite subset of $\lambda=\{\mu \mid \mu<\lambda\}, I(\Phi)$ is defined as follows. If $\Phi=\left\{\sigma_{1}, \cdots, \sigma_{m}\right\}$ such that $\sigma_{1}<\sigma_{2}<\cdots<\sigma_{m}$, put $\sigma_{0}=0$ and $\sigma_{m+1}=\lambda$, and let

$$
I(\Phi)=\bigcap_{i=0}^{m} \operatorname{Ann}\left(p^{\sigma_{i}} G[p] / p^{\sigma_{i+1}} G[p]\right) .
$$

Theorem 1.4 will be an immediate consequence of Theorem 1.3 and the following result.

Corollary 2.2. Suppose that $J(R)=I(G)$ and let $I$ be an ideal of $R$. Then the following conditions are equivalent.

(i) I is quasi-regular.

(ii) The restriction of $I$ to $G[p]$ is nil.

(iii) The restriction of $I$ to $G[p]$ is locally nilpotent.

Proof. Obviously, (iii) implies (ii). By (2.1) of [3], (ii) implies (i). Assume (i). Then $I \subseteq J(R)=I(G)$, and Lemma 2.1 completes the proof.

For the next result, $G$ need not be reduced. For $X \subseteq G$ and $I \subseteq R, X I$ denotes the set of all $x \varepsilon$ where $x \in X$ and $\varepsilon \in I$.

LEMMA 2.3. For each integer $n \geqq 0, H(G) \cap A n n p^{n} G$ is a nilpotent ideal of $R$.

Proof. Let $I=H(G) \cap \operatorname{Ann} p^{n} G$. Clearly, $I$ is an ideal of $R$. Moreover,

$$
G[p] I^{n+1} \subseteq p^{n} G I=0,
$$

which implies

$$
G\left[p^{k}\right] I^{n+1} \cong G\left[p^{k-1}\right] \quad \text { for each } k \geqq 1 .
$$

Furthermore,

$$
G I \leqq G\left[p^{n}\right]
$$

Hence, using (2.4),

$$
G I^{1+(n+1) n} \cong G\left[p^{n}\right]^{(n+1) n} \subseteq G\left[p^{n-n}\right]=0,
$$

as desired.

The set $T(R)$ of all torsion elements of $R$ is an ideal of $R$ [1; p. 278] which, as an abelian group, is a $p$-group. Hence

$$
\begin{aligned}
T(R) & =\left\{\varepsilon \in R \mid p^{n} \varepsilon=0 \text { for some } n<\omega\right\} \\
& =\bigcup_{n<\omega} \operatorname{Ann} p^{n} G=\sum_{n<\omega} \operatorname{Ann} p^{n} G .
\end{aligned}
$$


COROLLARY 2.5. $H(G) \cap T(R)=\sum_{n<\omega}\left[H(G) \cap\right.$ Ann $\left.p^{n} G\right]$ is a locally nilpotent ideal contained in $N(R)$.

Proof of Theorem 1.5. We first show the equivalence of conditions (ii)-(vii). By definition of the various ideals, (ii) implies (vi), which, in turn, using (1.1), implies (v). That (v) implies (iv) and (iv) implies (iii) is obvious. Assume (iii). By (1.2), Ann $G[p] \subseteq$ $I(G) \subseteq J(G)$. Thus, (iii) implies that the multiplication $p \cdot 1_{G}$ is nilpotent. Consequently, $\left(p \cdot 1_{G}\right)^{n}=p^{n} \cdot 1_{G}=0$ for some integer $n \geqq 0$ and $p^{n} G=0$. Thus (vii) follows from (iii). Assume (vii). Then $p^{n} G=0$ for some integer $n \geqq 0$, and Ann $p^{n} G=R$. Thus

$$
H(G)=H(G) \cap R=H(G) \cap \operatorname{Ann} p^{n} G .
$$

Using Lemma 2.3, it follows that $H(G)$ is nilpotent and, observing (1.2), so is $J(R)$. Hence (vii) implies (ii), and the last six conditions are equivalent. Clearly, (ii) implies (i). The proof will be completed once we show that $N(R)$ is not nilpotent if $G$ is unbounded. Suppose the latter and pick an integer $n \geqq 1$. We construct $\varepsilon \in N(R)$ such that $\varepsilon^{n} \neq 0$. Since $G$ is unbounded and reduced, $G$ has a decomposition

$$
G=\bigoplus_{i=1}^{n+1}\left\langle a_{i}\right\rangle \oplus C
$$

where, for $i=1, \cdots, n$, the order of $a_{i}$, call it $m_{i}$, is strictly less than the order of $a_{i+1}$. Define $\varepsilon \in R$ by

$$
\begin{aligned}
& a_{\imath} \varepsilon=p^{m_{i+1}-m_{i}} a_{i+1}, \quad i=1, \cdots, n \\
& a_{n+1} \varepsilon=0 \\
& C \varepsilon=0
\end{aligned}
$$

Since, for each $i \leqq n, m_{i+1}-m_{i} \geqq 1, \varepsilon \in H(G)$. Clearly,

$$
p^{m_{n+1}} G \varepsilon \subseteq C \varepsilon=0 \text {. }
$$

Hence $\varepsilon \in H(G) \cap \operatorname{Ann} p^{m_{n+1} G}$ and $\varepsilon \in N(R)$, by Corollary 2.5. One easily verifies that

$$
a_{1} \varepsilon^{n}=p^{m_{n+1}-m_{1}} a_{n+1} \neq 0 .
$$

Hence $\varepsilon^{n} \neq 0$ as desired.

LeMma 2.6. If $p^{\omega} G=0$ then

$$
I(G)=[H(G) \cap T(R)]+\operatorname{Ann} G[p] .
$$

Proof. Since Ann $G[p] \subseteq I(G)$, it suffices to show that, for each 
$\varepsilon \in I(G)$, there exists $\eta \in H(G) \cap T(R)$ so that $\varepsilon$ and $\eta$ coincide on $G[p]$. Let $\varepsilon \in I(G)$. By definition of $I(G), p^{\omega} G=0$ implies the existence of $m<\omega$ such that $p^{m} G[p] \varepsilon=0$. It is will known that $G$ has a decomposition

$$
G=A \oplus B, \quad p^{m} G=p^{m} B \supseteq B[p] .
$$

Define $\eta \in R$ by

$$
\begin{aligned}
& B \eta=0 \\
& a \eta=a \varepsilon \text { for all } \quad a \in A .
\end{aligned}
$$

Since $A(\eta-\varepsilon)=0$ and

$$
B[p] \varepsilon \subseteq p^{m} G[p] \varepsilon=0=B \eta,
$$

$\varepsilon$ and $\eta$ agree on $G[p]$. Thus, $\varepsilon \in I(G)$ implies $\eta \in I(G) \subseteq H(G)$. By construction, $p^{m} G \eta \cong B \eta=0$, hence $p^{m} \eta=0$ and $\eta \in T(R)$, completing the proof.

Observing (1.1), the following corollary together with Theorem 1.3 immediately yields Theorem 1.6.

Corollary 2.7. Suppose that $p^{\omega} G=0$ and $J(R)=I(G)$. Then

$$
J(R)=N(R)+\operatorname{Ann} G[p] .
$$

Proof. Lemma 2.6, Corollary 2.5 and (1.1).

Only Theorem 1.3 remains to be proven. For this we require the following observation.

LEMmA 2.8. Let $G=P \oplus C$ and let $\iota: P \rightarrow G$ and $\pi: G \rightarrow P$ be the corresponding canonical injection and projection respectively. Then, if $J$ is a quasi-regular ideal of End $G$, the set

$$
\iota J \pi=\{\varepsilon \varepsilon \pi \mid \varepsilon \in J\}
$$

is a quasi-regular ideal of End $P$.

Proof. Define $f:$ End $G \rightarrow$ End $P$ by $f(\varepsilon)=\iota \varepsilon \pi$ for all $\varepsilon \in$ End $G$. Then $f$ is a surjective ring homomorphism [1; p. 217]. Such functions map quasi-regular ideals to quasi-regular ideals [4; p. 8].

From now on we assume that $G$ is sufficiently projective. As above, $p^{\lambda} G=0$.

Proposition 2.9. Let $J$ be a quasi-regular ideal End G. Then, for each ordinal $\sigma<\lambda, p^{\sigma} G[p] J \subseteq p^{\sigma+1} G$. 
Proof. Let $\varepsilon \in J$ and let $x \in p^{\sigma} G[p]$. By hypothesis, $G$ has a decomposition

$$
G=P \oplus C \text { where } x, x \varepsilon \in P, P \text { totally projective. }
$$

Let $\iota$ and $\pi$ be as in Lemma 2.8. Then

$$
x \varepsilon=x \iota \varepsilon \pi \in\left(p^{\sigma} G[p] \cap P\right)(\iota \varepsilon \pi)=p^{\sigma} P[p](\iota \varepsilon \pi) \subseteq\left(p^{\sigma} P[p]\right)(\iota J \pi) .
$$

By Lemma 2.8, $\iota J \pi$ is a quasi-regular ideal of End $P$ and, since $P$ is totally projective, (2.2) of [3] implies $\left(p^{\sigma} P[p]\right)(\iota J \pi) \subseteq p^{\sigma+1} P$. Hence,

$$
x \varepsilon \in p^{\sigma+1} P \subseteq p^{\sigma+1} G,
$$

completing the proof.

For $x \in G$, let $h(x)$ denote the (possibly transfinite) height of $x$.

Proposition 2.10. Let $J$ be a quasi-regular ideal of End $G$, let $\tau$ be an ordinal such that $0<\tau \leqq \lambda$, and let $\varepsilon \in J$, then there exists an ordinal $\sigma<\tau$ such that $p^{\sigma} G[p] \varepsilon \subseteq p^{\tau} G$.

Proof. Assume, by way of contradiction, that $p^{\sigma} G[p] \varepsilon \nsubseteq p^{\tau} G$ for all $\sigma<\tau$. Then, for each $\sigma<\tau$, there exists $x_{\sigma} \in p^{\sigma} G[p]$ such that $x_{\sigma} \varepsilon \notin p^{\tau} G$ and thus, by Proposition 2.9, $\sigma \leqq h\left(x_{\sigma}\right)<h\left(x_{\sigma} \varepsilon\right)<\tau$. The same result implies that $\tau$ is a limit ordinal. Consequently, there exist $y_{i} \in G[p], i=0,1,2, \cdots$, such that

$$
h\left(y_{i}\right)<h\left(y_{i} \varepsilon\right)<h\left(y_{i+1}\right)<\tau \text { for } i=0,1,2, \cdots .
$$

Let

$$
\rho=\sup \left\{h\left(y_{i}\right) \mid i=0,1,2, \cdots\right\} .
$$

Since $G$ is sufficiently projective, it has a decomposition

$$
\left\{\begin{array}{l}
G=P \oplus C, P \text { totally projective, } \\
y_{i}, y_{i} \varepsilon \in P \text { for } i=0,1,2, \cdots
\end{array}\right.
$$

By (2.12), $P$ has length at least $\rho$. Let $\iota$ and $\pi$ be the maps of Lemma 2.8. Then $\iota \varepsilon \pi \in \iota J \pi$ which, by Lemma 2.8 , is a quasi-regular ideal of End $P$. Since $P$ is totally projective, Theorem 3.5 of [3] implies the existence of an ordinal $\sigma<\rho$ such that

$$
p^{\sigma} P[p](\iota \varepsilon \pi) \subseteq p^{\rho} P \text {. }
$$

By (2.11) and (2.12), there exists an $i$ such that

$$
\sigma \leqq h\left(y_{i}\right)<\rho,
$$

and (2.14), together with (2.13), implies 


$$
y_{i} \varepsilon=y_{i} \varepsilon \varepsilon \pi \in p^{\sigma} P[p](\varepsilon \varepsilon \pi) \subseteq p_{\rho} P .
$$

Hence, using (2.11), $\rho \leqq h\left(y_{i} \varepsilon\right)<h\left(y_{i+1}\right)$. This contradiction to (2.14) completes the proof.

Proof of Theorem 1.3. By (1.2), it suffices to show that $J($ End $G) \cong I(G)$. Let $\varepsilon \in J($ End $G)$. Proposition 2.10 implies the existence of $\gamma<\lambda$ such that $p^{r} G[p] \varepsilon \subseteq p^{\lambda} G=0$. Apply Proposition 2.10 repeatedly and use the fact that every properly descending sequence of ordinal numbers terminates after finitely many steps [10; p. 270].

\section{REFERENCES}

1. L. Fuchs, Infinite Abelian Groups, Vol. II, Academic Press, New York, 1973.

2. F. Haimo, Endomorphism radicals which characterize some divisible groups, Ann. Univ. Sci. Budapest, 10 (1967), 25-29.

3. J. Hausen, Quasi-regular ideals of some endomorphism rings, Illinois J. Math., 22 (1977), 845-851.

4. N. Jacobson, Structure of Rings, Amer. Math. Soc. Colloq. Publ., Vol. 37, Revised Edition, Providence, 1968.

5. A. G. Kuroš, Radicals of rings and algebras, Colloquia Mathematica Societatis János Bolyai, Rings Modules and Radicals, Keszthely (Hungary), (1971), 297-314.

6. M. Le Borgne, Groupes ג-separables, C. R. Acad. Sc. Paris 281 (Serie A) (1975), 415-417.

7. W. Liebert, The Jacobson radical of some endomorphism rings, J. Reine Angew. Math., 262/263 (1973), 166-170.

8. C. Megibben, A generalization of the classical theory of primary groups, Tôhoku Math. J., 22 (1970), 347-356.

9. R. S. Pierce, Homomorphisms of primary abelian groups, Topics in Abelian Groups, Chicago, 1963, pp. 215-310.

10. W. Sierpinski, Cardinal and Ordinal Numbers, Warszawa, 1958.

11. E. A. Walker, The groups $P_{\beta}$, Symposia Math., Vol. 13, Academic Press, New York, (1974), 245-255.

12. K. D. Wallace, $C_{\lambda}$-groups and $\lambda$-basic subgroups, Pacific J. Math., 43 (1972), 799-809.

13. R. B. Warfield, Jr., Classification theorems for p-groups and modules over a discrete valuation rings, Bull. Amer. Math. Soc., 78 (1972), 88-92.

14. - A classification theorem for abelian p-groups, Trans, Amer. Math. Soc., 210 (1975), 149-168.

Received May 3, 1977. Research by the first author was supported in part by a grant from the University of Houston.

UNIVERSITY OF HOUSTON

HOUSTON, TX 77004 


\section{PACIFIC JOURNAL OF MATHEMATICS}

\section{EDITORS}

RICHARD ARENS (Managing Editor)

University of California

Los Angeles, CA 90024

Charles W. Curtis

University of Oregon

Eugene, OR 97403

C. C. Moore

University of California

Berkeley, CA 94720

\section{J. DugundJI}

Department of Mathematics

University of Southern California

Los Angeles, CA 90007

R. FInN and J. Milgram

Stanford University

Stanford, CA 94305

\section{ASSOCIATE EDITORS}
E. F. BECKENBACH
B. H. NeumanN
F. WOLF
K. YoSHIDA

\section{SUPPORTING INSTITUTIONS}

\author{
UNIVERSITY OF BRITISH COLUMBIA \\ CALIFORNIA INSTITUTE OF TECHNOLOGY \\ UNIVERSITY OF CALIFORNIA \\ MONTANA STATE UNIVERSITY \\ UNIVERSITY OF NEVADA, RENO \\ NEW MEXICO STATE UNIVERSITY \\ OREGON STATE UNIVERSITY \\ UNIVERSITY OF OREGON
}

\author{
UNIVERSITY OF SOUTHERN CALIFORNIA \\ STANFORD UNIVERSITY \\ UNIVERSITY OF HAWAII \\ UNIVERSITY OF TOKYO \\ UNIVERSITY OF UTAH \\ WASHINGTON STATE UNIVERSITY \\ UNIVERSITY OF WASHINGTON
}

The Supporting Institutions listed above contribute to the cost of publication of this Journal, but they are not owners or publishers and have no responsibility for its content or policies.

Mathematical papers intended for publication in the Pacific Journal of Mathematics should be in typed form or offset-reproduced, (not dittoed), double spaced with large margins. Please do not use built up fractions in the text of the manuscript. However, you may use them in the displayed equations. Underline Greek letters in red, German in green, and script in blue. The first paragraph or two must be capable of being used separately as a synopsis of the entire paper. Items of the bibliography should not be cited there unless absolutely necessary, in which case they must be identified by author and journal, rather than by item number. Manuscripts, in triplicate, may be sent to any one of the editors. Please classify according to the scheme of Math. Reviews, Index to Vol. 39. All other communications should be addressed to the managing editor, or Elaine Barth, University of California, Los Angeles, California, 90024.

50 reprints to each author are provided free for each article, only if page charges have been substantially paid. Additional copies may be obtained at cost in multiples of 50 .

The Pacific Journal of Mathematics is issued monthly as of January 1966. Regular subscription rate: $\$ 72.00$ a year (6 Vols., 12 issues). Special rate: $\$ 36.00$ a year to individual members of supporting institutions.

Subscriptions, orders for numbers issued in the last three calendar years, and changes of address should be sent to Pacific Journal of Mathematics, 103 Highland Boulevard, Berkeley, California, 94708. Older back numbers obtainable from Kraus Periodicals Co., Route 100, Millwood, NY 10546.

PUBLISHED BY PACIFIC JOURNAL OF MATHEMATICS, A NON-PROFIT CORPORATION

Printed at Kokusai Bunken Insatsusha (International Academic Printing Co., Ltd.). 8-8, 3-chome, Takadanobaba, Shinjuku-ku, Tokyo 160, Japan.

Copyright (C) 1978 by Pacific Journal of Mathematics

Manufactured and first issued in Japan 


\section{Pacific Journal of Mathematics

Vol. 74, No. $2 \quad$ June, 1978

Aharon Atzmon, Spectral synthesis in some spaces of bounded continuous

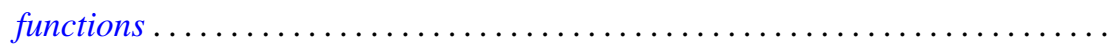

Karl Egil Aubert and Isidor Fleischer, Tensor products of ideal systems and their modules.............................................

Richard F. Basener, Several dimensional properties of the spectrum of a uniform

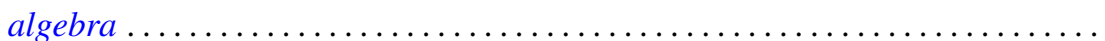

R. H. Bing and Michael Peter Starbird, Super triangulations ............. 307

Andrew Carson, Coherent polynomial rings over regular rings of finite index .....

Robert M. DeVos and Frederick W. Hartmann, Sequences of bounded summability domains .................................................

George Grätzer and R. Padmanabhan, Symmetric difference in abelian groups ....

Robert L. Griess, Jr., A remark about groups of characteristic 2-type and

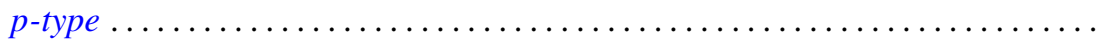

Emil Grosswald and F. J. Schnitzer, A class of modified $\zeta$ and L-functions........

Jutta Hausen and Johnny Albert Johnson, Ideals and radicals of some

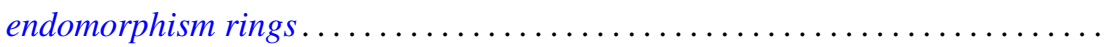

Jean Ann Larson, A solution for scattered order types of a problem of Hagendorf ............................................

Peter A. McCoy, Extremal properties of real biaxially symmetric potentials in $E^{2(\alpha+\beta+2)}$

Héctor Alfredo Merklen, Hereditary crossed product orders .

Hal G. Moore and Adil Mohamed Yaqub, Equational definability of addition in certain rings...

Robert Laurens Moore, Reductivity in $C^{*}$-algebras and essentially reductive operators. . .

Joseph Alvin Neisendorfer, Lie algebras, coalgebras and rational homotopy theory for nilpotent spaces...

William Raymond Nico, Bounded monoids

Richard Paul Osborne, Simplifying spines of 3-manifolds ...

Richard Paul Osborne, The simplest closed 3-manifolds. With an appendix by Osborne and J. Yelle.

Clayton Collier Sherman, The $K$-theory of an equicharacteristic discrete valuation ring injects into the $K$-theory of its field of quotients.... .

Mitchell Herbert Taibleson, The failure of even conjugate characterizations of $H^{1}$

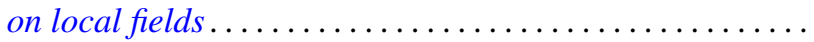

Keti Tenenblat, On characteristic hypersurfaces of submanifolds in Euclidean space ...................................

Jeffrey L. Tollefson, Involutions of Seifert fiber spaces..........

Joel Larry Weiner, An inequality involving the length, curvature, and torsions of a curve in Euclidean $n$-space .......................

Neyamat Zaheer, On generalized polars of the product of abstract homogeneous polynomials.... 